\title{
Evaluation of an Automated, High Throughput Flowcytometry Based Technique for Diagnosis of Malaria
}

\author{
Arti Negi ${ }^{1}$, Bijay Ranjan Mirdha ${ }^{2}$, S. Arulselvi ${ }^{3}$, Avinash Singh ${ }^{4}$, Sangram Singh Patel ${ }^{1}$, \\ Anjali Agarwal ${ }^{1}$ and Jyotish C. Samantaray ${ }^{2}$ \\ ${ }^{1}$ Department of Microbiology, Hind Institute of Medical Sciences, Barabanki, \\ Uttar Pradesh, India \\ ${ }^{2}$ AIIMS, Department of Microbiology, Ansari Nagar, New Delhi, India \\ ${ }^{3}$ Department of Lab Medicine, Jai Prakash Narayan Apex Trauma Centre, AIIMS, \\ New Delhi, India \\ ${ }^{4}$ Department of Microbiology, Sanjay Gandhi Post Graduate Institute of Medical Sciences \\ (SGPGIMS), Lucknow, Uttar Pradesh, India \\ *Corresponding author
}

\section{A B S T R A C T}

Malaria is a potential medical emergency which should be treated as early as possible. It has become a leading cause of morbidity and mortality in many countries due to delay in diagnosis and treatment. Limitations of malaria diagnosis using classical methods have led to the development of several new techniques that simplify and speed up the diagnosis and

\section{Keywords}

Malaria, Sysmex 2100, Haematology analyser, Scattergram, Flowcytometry

Article Info

Accepted: 06 May 2018 Available Online: 10 June 2018 increases the sensitivity. In the past few years, a newer technique for diagnosis of malaria has emerged based on flowcytometry. We conducted a study where we compared conventional methods of malaria diagnosis, microscopy [Giemsa, Acridine Orange, QBC assay (quantitative buffy coat)], antigen detection (pLDH) with flowcytometry based haematology analyser.A total of three hundred twenty five $(n=325)$ clinically suspected malaria patients were enrolled in the study, each sample $(n=325)$ was examined for the presence of malaria parasites by both conventional methods and flowcytometry and results were compared. The scattergram formed by five parameter counts i.e., lymphocytes, monocytes, eosinophills, neutrophils and basophils by haematology analyser were interpreted and analysed. It is capable of detecting specific abnormalities in the blood of patients suffering from malaria in the form of abnormal scattergrams. Based on abnormal scattergram in the DIFF plot, the overall sensitivity and specificity of flowcytometry based hematology analyser came out to be $78.46 \%$ and $90 \%$ respectively, which was comparable to the Giemsa, a routine test done in many laboratories. Thus we conclude, flowcytometry based 5 parts differential haematology analyser is a rapid, automated, and high throughput device for early detection of malaria, especially in unsuspected cases.

\section{Introduction}

Malaria is the one of the most important infectious disease, both in tropical and subtropical regions, and continues to be a major global health problem, with over $40 \%$ of the world's population exposed to varying degrees of malaria risk in some 100 countries 
(Tangpukdee et al., 2009). India accounts for nearly $6 \%$ of global malaria burden, where about $95 \%$ population resides in malaria endemic areas (WHO, World Malaria Report 2017). Although effective ways to manage malaria now exist, but still the number of malaria cases and malaria related complications are on rise. In this emergency situation, prompt and accurate diagnosis plays a key role for effective management of malaria.

Routinely, malaria is diagnosed using a combination of clinical observations, case history, and diagnostic tests, principally microscopic examination of Giemsa stained slides. However, classical microscopic examination is labour intensive, time consuming and have low sensitivity. Limitations of malaria diagnosis using conventional methods have led to the development of several new, adjuvant and alternative techniques including automation (e.g., polymerase chain reaction) that simplify and speed up the diagnosis and increases the sensitivity (Hanscheid et al., 1999; Zalis et al., 1996).

For the past few years, there is growing interest in the use of haematology analysers for the presumptive diagnosis and screening of malaria infection, along with haematological parameters (Hanscheid et al., 2000). These analysers work on the principle of flowcytometry and measure the different blood corpuscular elements, using a semiconductor laser and produce various scattergrams on the monitor. Abnormal white blood cell (WBC) scattergrams were found in many malaria-infected patients, which could help in early detection of malaria, as it has been reported that abnormal depolarizing patterns (scattergrams) correlated very well with high sensitivity for malaria diagnosis (Huh et al., 2005; Hanscheid et al., 2001). The major advantage of this method is its potential to detect malaria even in those cases where clinical suspicion does not lead to a request for a malaria test unlike conventional methods (Hanscheid et al., 1999). Keeping all these advantages of flowcytometry based hematology analysers in mind, we conducted a study where the conventional methods of malaria diagnosis, microscopy [Giemsa, Acridine Orange, QBC assay (quantitative buffy coat)], antigen detection ( $\mathrm{pLDH})$ were compared with flowcytometry based haematology analyser. The main aim of the study was to evaluate the automated flowcytometry based technique for the diagnosis of malaria and further evaluation of the sensitivity, specificity and other performance parameters of flowcytometry in comparison to conventional methods (Giemsa, AO, QBC, Ag detection).

\section{Materials and Methods}

This prospective study was conducted in the Department of Microbiology, in collaboration with Department of Laboratory Medicine (Jai Prakash Narayan (JPN), Apex Trauma Centre) of All India Institute of Medical Sciences, New Delhi over a period of two years. A total of three hundred twenty five $(n=325)$ clinically suspected malaria patients were enrolled in the study. Proper patient consent was taken at the time of sample collection. Around $3 \mathrm{ml}$ of blood sample was taken in EDTA vial to perform various tests. Each sample $(n=325)$ was examined for the presence of malaria parasites by both Giemsa and Acridine Orange (AO) staining methods, Quantitative buffy coat (QBC) assay [Clay Adams Divisions, Becton Dickinson and Company, Franklin Park New Jersey, USA] and pLDH, (parasite Lactate Dehydrogenase, Advantage Mal Card, Mitra \& Co Pvt. Ltd, New Delhi, India) antigen detection as per manufacturer's guidelines. Parasite count was done by both Giemsa and Acridine Orange staining methods. All the blood smears, QBC 
assay, antigen detection and flowcytometry scattergrams were examined independently by two observers and findings were confirmed by a senior microbiologist. Flowchart showing sample processing in haematology analyser (Figure 1).

\section{Figure 1: Flowchart showing sample processing in haematology analyser}

Sample was kept in the haematology analyser

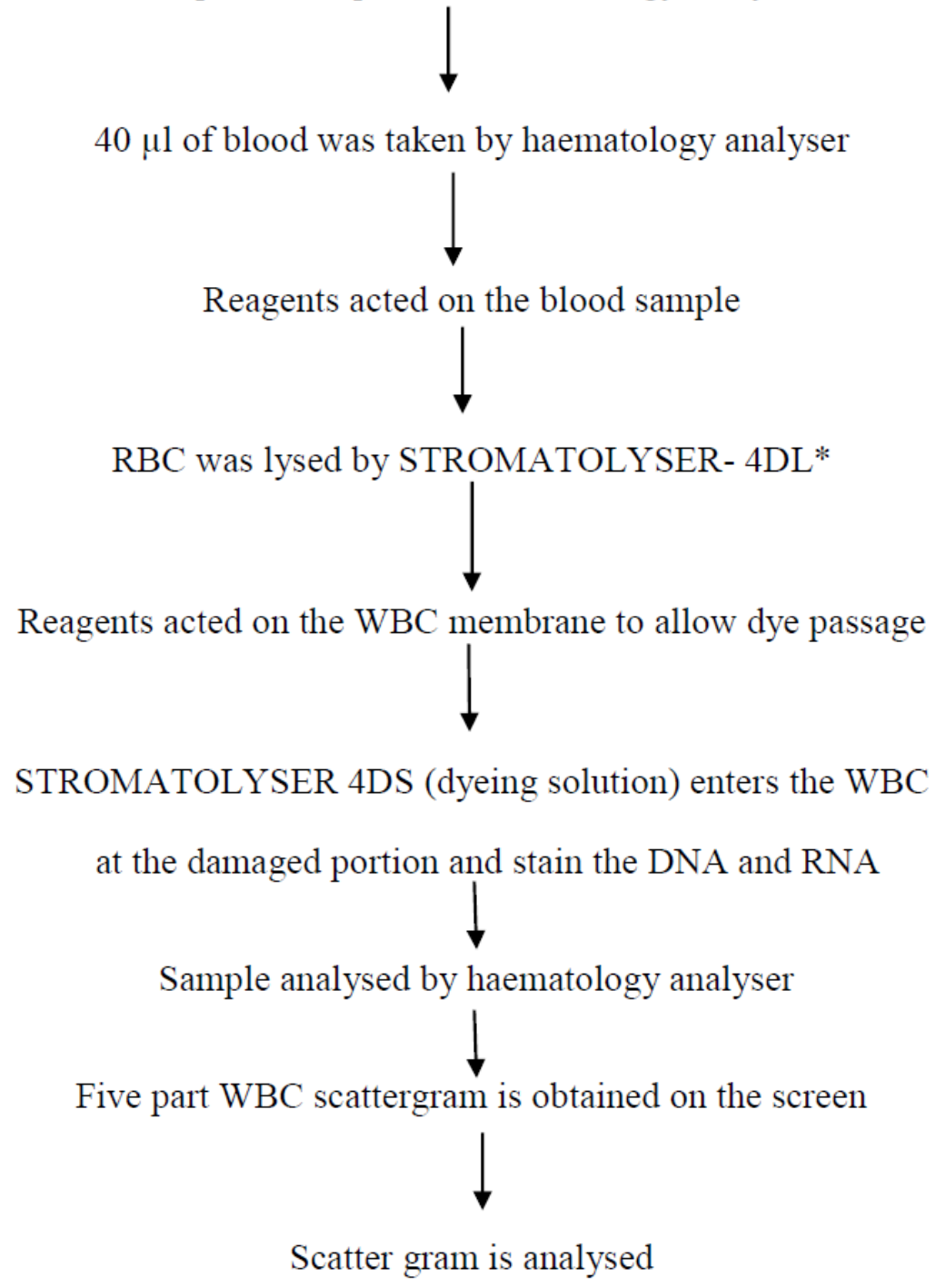

*RBC were lysed with acid haemolytic reagent, STROMATOLYSER-4DL

In the present study, we have used 5 part DIFF (differential) hematology analyser, Sysmex XE-2100 (Sysmex Corporation, Kobe, Kansai, Japan) which works on the principle of flowcytometry and measures the different blood corpuscular elements, using a semiconductor laser. This instrument differentiates WBCs mainly on the basis of side fluorescence and side-scattered light by measuring DNA and RNA content of the cell stained with fluorescent dye (polymethine dye) and granularity of the cell and side 
fluorescence measures respectively. The normal scattergram in the DIFF plot is constituted of five parameters; lymphocytes (pink), monocytes (green), neutrophils + basophil (blue), eosinophils (red) with a definitive space between the neutrophil and eosinophil populations (Figure 2).

A malaria infected RBCs with various parasitic morphologic forms (trophozoites, schizonts, gametocytes) and hemozoin pigment containing cells (monocytes and neutrophils) mimic the above-described patterns and exhibit various abnormal WBC scattergram during routine Complete Blood Count (CBC) analysis as shown in Figure 2. The scattergram formed by five parameter counts (lymphocytes, monocytes, eosinophils, neutrophils and basophils) were interpreted and analysed.

Out of $325,65[65 / 325(20 \%)]$ confirmed cases of malaria positive by microscopy and /or antigen detection were taken as study group (positive case of malaria). 260 clinically suspected cases out of 325[260/325 $(80 \%)$ ], which were negative by conventional assay for malaria were taken as control group as per biostatistician's advice. All of these control groups were confirmed negative either by microscopy or /and antigen detection.

Statistical analysis of all the methods was calculated by $2 \times 2$ contingency table analysis.

\section{Results and Discussion}

Out of 325 clinically suspected cases, 65 samples were found to be positive for malaria by conventional methods. $P$. vivax $[46 / 65$ $(70.8 \%)$ ] was the predominant species followed by $P$. falciparum [19/65 (29.3\%)].
Among 325 clinically suspected malaria patients, 208 were males and 117 were females and in 65 malaria positive cases 44 were males and 21 were females. Within the study population, majority of males $(40 \%)$ and females $(18.5 \%)$ belonged to the age group of 20-50 years, suggesting that malaria is more common in this age group among male population. A seasonal pattern of distribution of malaria was observed in our study. It was observed that the highest number of malaria cases occurred during the month from July to October.

A maximum number of malaria positive cases were detected by QBC (65/65), followed by antigen detection pLDH (63/65), AO (58/65), Giemsa (53/65) and Sysmex-2100 (51/65) respectively (Table 1). QBC was found to be $100 \%$ sensitive and specific, followed by antigen detection, AO, Giemsa and flowcytometry respectively as shown in Table 1 .

Out of the 65 confirmed malaria positive cases, 51 samples showed various abnormal patterns in DIFF scattergram such as extended neutrophil, double neutrophil, grey zone, double eosinophil and blue zone on flowcytometric analyser (Figure 2). Extended neutrophil was the commonest pattern observed (39.2\%) followed by the double neutrophil (29.41\%) (Table 2). Fourteen malaria positive samples showed normal scattergram on haematology analyser (21.5 $\%)$. In control group, majority 234 (90\%) had normal scattergram pattern in flowcytometry. Among 26 (10\%) abnormal pattern formed in malaria negative cases, the most common pattern seen was grey zone [12/26 (46.15\%)] as shown in Table 3. 


\section{Figure 2: Diff Scattergrams of Sysmex 2100}

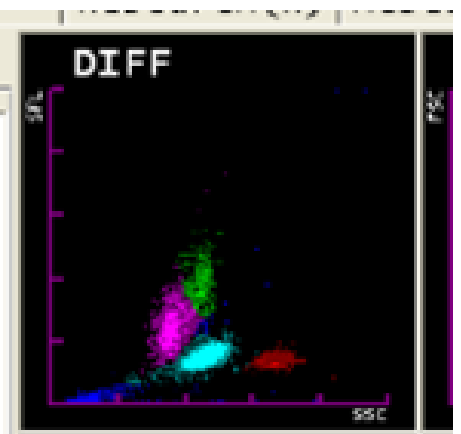

1) Normal Scattergram

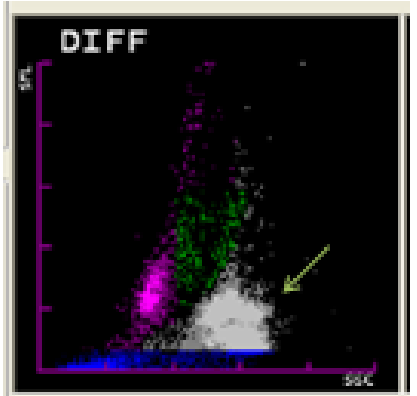

4) Grey zone

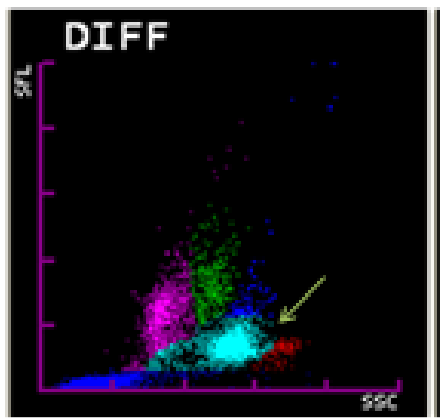

2) Extended Neutrophil

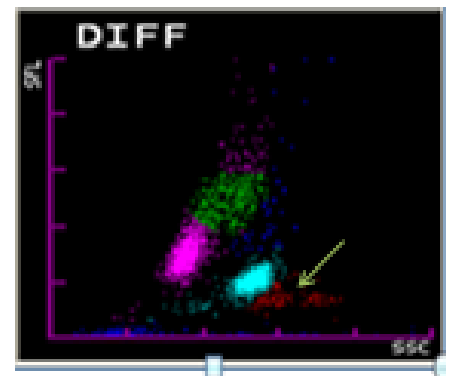

5) Double eosinophil

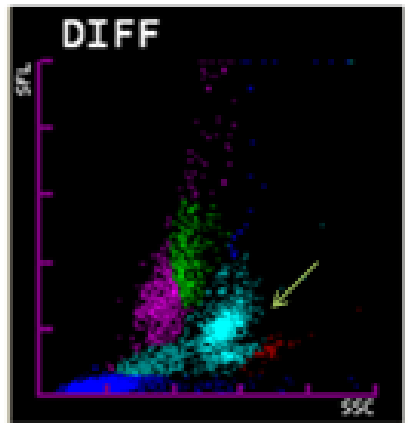

3) Double neutrophil

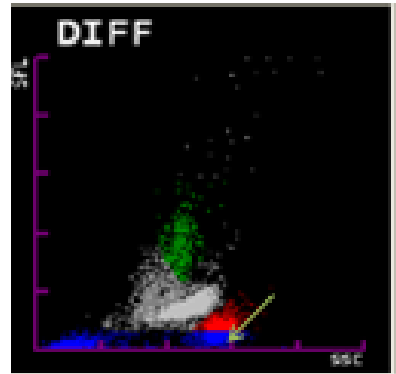

6) Blue zone

Table.1 Comparison of Detection of Malaria Parasite by Conventional Methods and FlowCytometric Analyser (Sysmex 2100)

\begin{tabular}{|c|c|c|c|c|c|c|}
\hline Method & $\begin{array}{c}\text { Positive } \\
n=65\end{array}$ & $\begin{array}{c}\text { Negative } \\
n=260\end{array}$ & $\begin{array}{l}\text { Sensitivity } \\
(\%)\end{array}$ & $\begin{array}{l}\text { Specificity } \\
(\%)\end{array}$ & $\begin{array}{l}\text { PPV\% } \\
(95 \% \text { CI })\end{array}$ & $\begin{array}{l}\text { NPV\% } \\
(95 \% C I)\end{array}$ \\
\hline GIEMSA & 53 & 260 & 81.53 & 100 & $\begin{array}{c}100(93.24- \\
100)\end{array}$ & $\begin{array}{c}95.59(92.45- \\
97.46)\end{array}$ \\
\hline $\mathrm{AO}$ & 58 & 260 & 89.23 & 100 & $\begin{array}{c}100(93.79- \\
100)\end{array}$ & $\begin{array}{c}97.38 \% \\
(94.69-98.72)\end{array}$ \\
\hline QBC & 65 & 260 & 100 & 100 & $\begin{array}{c}100(94.42- \\
100)\end{array}$ & $\begin{array}{c}100(98.54- \\
100)\end{array}$ \\
\hline $\begin{array}{l}\mathrm{Ag} \\
\text { DETECTION }\end{array}$ & 63 & 260 & 96.92 & 100 & $\begin{array}{c}100(94.25- \\
100)\end{array}$ & $\begin{array}{l}99.24(97.26- \\
99.79)\end{array}$ \\
\hline $\begin{array}{l}\text { FLOWCYTO } \\
\text { METRY } \\
\text { SYSMEX-2100 }\end{array}$ & 51 & 234 & 78.46 & 90 & $\begin{array}{c}66.23(55.1 \\
2-75.8)\end{array}$ & $\begin{array}{c}94.35(90.75 \\
-96.61)\end{array}$ \\
\hline
\end{tabular}

PPV: Positive Predictive Value NPV: Negative Predictive Value 


\begin{tabular}{|c|c|}
\hline \multicolumn{2}{|c|}{ Table.2 Types of Scattergram Patterns Seen In Malaria Positive Cases (n=65) } \\
\hline Types of scattergram pattern & Number (Percentage\%) \\
A)ABNORMAL PATTERNS & $51(78.46 \%)$ \\
\hline 1. Extended Neutrophil & $20(39.2 \%)$ \\
\hline 2. Double Neutrophil & $15(29.41 \%)$ \\
\hline 3. Grey Zone & $9(17.64 \%)$ \\
\hline 4. Double Eosinophil & $2(3.92 \%)$ \\
\hline 5. Blue Zone & $5(9.8 \%)$ \\
\hline B) NORMAL PATTERN & $14(21.535 \%)$ \\
\hline TOTAL & 65 \\
\hline
\end{tabular}

Table.3 Types of Abnormal Scattergram Patterns Seen in Control Group (Malaria Negative) $(n=260)$

\begin{tabular}{|c|c|}
\hline Types of scattergram & Numbers \\
\hline A) No abnormal zone & 234 \\
\hline B) Abnormal patterns & 2 \\
a. Extended Neutrophil & 7 \\
b. Double Neutrophil & $\mathbf{1 2}$ \\
c. Grey Zone & 2 \\
d. Double Eosinophil & 3 \\
e. Blue Zone & \\
\hline
\end{tabular}


Table.4a Comparison of detection of malaria parasite by conventional methods and Flowcytometric analyser in case of parasite count $2-200 / \mu \mathrm{l}(\mathrm{n}=24)$ (in malaria positive cases)

\begin{tabular}{|c|c|c|c|}
\hline Method & $\begin{array}{c}\text { Positive } \\
\mathbf{n = 2 4}\end{array}$ & Negative & $\begin{array}{c}\text { Sensitivity } \\
\mathbf{\%}\end{array}$ \\
\hline GIEMSA & 13 & 11 & 54.16 \\
\hline AO & 17 & 7 & 70.83 \\
\hline QBC & 24 & 0 & 100 \\
\hline Ag DETECTION & 22 & 2 & 92 \\
\hline Flow-cytometry & $\mathbf{1 0}$ & $\mathbf{1 4}$ & $\mathbf{4 1 . 6 6}$ \\
\hline
\end{tabular}

Table.4b Comparison of detection of malaria parasite by conventional methods and Flowcytometric analyser in case of parasite count $>200 / \mu 1$ $(n=41)$ (in malaria positive cases)

\begin{tabular}{|c|c|c|c|}
\hline Method & POSITIVE & NEGATIVE & $\begin{array}{c}\text { SENSITIVITY } \\
\mathbf{\%}\end{array}$ \\
\hline GIEMSA & 40 & 1 & 97.56 \\
\hline AO & 41 & 0 & 100 \\
\hline QBC & 41 & 0 & 100 \\
\hline Ag DETECTION & 41 & 0 & 100 \\
\hline Flow-cytometry & 41 & 0 & $\mathbf{1 0 0}$ \\
\hline
\end{tabular}

\begin{tabular}{|l|l|l|l|l|}
\hline \multicolumn{5}{|c|}{ Table.5 Comparison of various techniques used for malaria diagnosis } \\
\hline METHODS & $\begin{array}{l}* * \text { TIME } \\
\text { REQUIRED }\end{array}$ & $\begin{array}{l}\text { SKILL } \\
\text { REQUIRED }\end{array}$ & $\begin{array}{l}\text { FIELD } \\
\text { APPLICABILITY }\end{array}$ & COST \\
\hline Giemsa (thin) & $30-60$ & Skilled & Yes & Low \\
\hline AO & $1-10$ & Skilled & Yes & Low \\
\hline pLDH test & $10-20$ & Semiskilled & Yes & High \\
\hline QBC assay & $10-15$ & Skilled & Yes & High \\
\hline PCR & $240-360$ & Skilled & No & Low \\
\hline $\begin{array}{l}\text { Hematology } \\
\text { analyser }\end{array}$ & $<1$ & Semiskilled & Yes & \\
\hline
\end{tabular}

**Sample processing-reporting in minutes.

Thus, based on these abnormal scattergram in the DIFF plot, the overall sensitivity and specificity of flowcytometry based hematology analyser came out to be $78.46 \%$ and $90 \%$ respectively (Table 1 ). We also evaluated the sensitivity, specificity and other performance parameters of flowcytometry in comparison to conventional methods in two 
different scenario i.e., when parasite count was $<200 / \mu \mathrm{L}$ ( 24 positive cases) and $>200 /$ $\mu \mathrm{L}$ (41 positive cases). Hematology analyser was unable to detect any abnormality in 14 malaria positive samples where parasite count was $<200 / \mu \mathrm{L}$ and gave normal scattergram, sensitivity was only $41.66 \%$ whereas in samples where parasite count was $>200 / \mu \mathrm{L}$ hematology analyser gave abnormal scattergram in all the positive samples and showed sensitivity of $100 \%$ as shown in Table $4 \mathrm{a}$ and $4 \mathrm{~b}$.

The need for improved and accurate malaria diagnosis has long been recognized. Local diagnostic modalities available may vary markedly and it is unlikely that a single diagnostic test would be ideal for all the situations. Recognition of the limitations of diagnosing malaria by light microscopy of Giemsa stained smears has led to the development of several new techniques that aim to simplify and speed up diagnosis and increase the sensitivity (Hänscheid et al., 1999). Good results have been obtained using fluorescent dyes like QBC (Levine et al., 1989) and simple dipstick tests to detect various antigens [WHO, 1996]. In recent years, PCR has also been added to the list and some authors regard it as new reference method, because of its superior sensitivity and specificity (Snounou et al., 1993). However, all these tests have the inherent disadvantage that they have to be specifically requested by an alert clinician who suspects the presence of malaria. Absence of this suspicion due to atypical or unsuspected clinical features in many patients is a main reason for high numbers of misdiagnoses (Kain et al., 1998).

In the past few years, a newer technique for diagnosis of malaria has emerged based on flowcytometry. There is substantial evidence that modern haematology analysers can detect malaria-associated events/flags, mostly by interference with the flowcytometry and direct current measurements (Briggs et al., 2006).

The present study evaluated 5 part DIFF hematology analyser based on flowcytometry for detection of malaria from patient's blood samples. The results were analyzed by comparison with the microscopic examination (Giemsa, AO, QBC) and antigen detection (pLDH). In our study, $P$. vivax [46/65 $(70.7 \%)]$ was the predominant species followed by $P$. falciparum [19/65 (29.3\%)], which was in concordance with the data provided by National Vector Borne Disease Control Programme at the website [www.nvbdcp.gov.in].

The present study reports the maximum cases of malaria in the rainy season which corroborates with several other studies (Madhavan et al., 2001; Thomson et al., 2005; Enosolease et al., 2003). It is mainly due to increase in mosquito breeding and transmission during the rainy season. Therefore, we would like to suggest that personal and community anti- mosquito measures taken during this peak season will be helpful to decrease the number of malaria cases and its complications. Out of 325 clinically suspected malaria patients, 209 $(64.3 \%)$ were males and $116(35.7 \%)$ were females. In 65 confirmed cases of malaria, 44 $(67.7 \%)$ were males and $21(32.3 \%)$ were females, showing predominance of malaria in males. The age distributions of 65 positive cases showed maximum positive cases in 2050 years of age group i.e., 38[38/65 (58.5\%)] out of which $26(68.42 \%)$ were males and 12 $(31.57 \%)$ were females. This could be due to the outdoor activity and mobility of male population in this particular age group for economic, educational and other reasons. Our observation on this aspect is in agreement with (Moore et al., 1995; Jamaiah et al., 2005). The present study also compared sensitivity, specificity and other performance 
parameters of hematology analyser with conventional methods and overall sensitivity and specificity of our study came out to be $78.46 \%$ and $90 \%$ respectively. The overall sensitivity of our study was more as compared to the study done by Huh et al., 2008, (69.4\% sensitivity and $100 \%$ specificity) but specificity of our study was low i.e. $90 \%$,as compared to previous literatures (Yoo et al., 2010; Campuzano-Zuluaga et al., 2010). A study conducted by Yoo et al., 2010 showed sensitivity and specifity of $46.2 \%$ and $99.7 \%$ respectively. The sensitivity of our study on hematology analyser was comparable to Giemsa staining, which is routinely used in many laboratories in India and other developing countries (Tangpukdee et al., 2009).

Most common abnormal scattergram pattern in our study was extended neutrophil $(39.2 \%)$ followed by double neutrophil (29.41\%) and least common pattern was double eosinophil (3.92\%), however a similar study conducted by Jungwon Huh et al., (2005) observed double eosinophil as the most common abnormal pattern. So far ours is the first study that has included different types of abnormal patterns and their frequencies.

The specificity (90\%) of our study was low as 26 malaria negative samples also gave abnormal scattergram i.e. false positive. Most common type of scattergram pattern in false positive samples was grey zone. Low specificity can be explained by storage problems and delay in haematology analysis. Ideally samples should be processed within 46 hours of withdrawing blood (Jonathan et al., 2001).

On evaluation of sensitivity, specificity and other performance parameters of flowcytometry in comparison to conventional methods in two different scenario i.e. when parasite count was $<200 / \mu \mathrm{L}$ (24 positive cases) and $>200 / \mu \mathrm{L}$ (41 positive cases), we observed that sensitivity of flowcytometry was high in cases where parasite count was > $200 / \mu \mathrm{L}$. In positive samples where parasite count was $<200 / \mu \mathrm{L},(24 / 65)$, hematology analyser was unable to detect any abnormality in 14/24 positive samples and gave normal scattergram pattern. The sensitivity came out to be only $41.66 \%$, while in samples of untreated cases where parasite count was > 200/ $\mu \mathrm{L}$ (41/65), hematology analyser gave abnormal scattergram in all the positive samples and showed sensitivity of $100 \%$.

The results were similar to study conducted by Hee Jin Huh et al., 2008 but their cut off parasite count was $500 / \mu \mathrm{L}$ instead of $200 /$ $\mu \mathrm{L}$. According to their study, sensitivity was $39.3 \%$ for the low parasitemia group when pseudoeosinophilia or an abnormal WBC scattergram was used for malaria detection, but this sensitivity increased significantly, to $88.6 \%$, for the high parasitemia group.This can be explained that maximum patients in our study, where parasite count was low $<200 / \mu \mathrm{L}$, were already on antimalarials while patients having parasite count $>200 / \mu \mathrm{L}$ were mostly untreated naive cases. So, decreased sensitivity of hematology analyser can be attributed to low parasite count, as in cases of prolonged treatment.

We also repeated flowcytometry in some patients who were undergoing treatment for malaria and followed them. It was observed that abnormal scattergram of malaria patients became normal after 48 to 72 hours of initiation of antimalarial treatment. The disappearance of parasitic forms in the follow-up blood samples corresponded with the resolution of abnormal pattern from the scattergram. So along with malaria detection flowcytometry based hematology analyser can also be used for monitoring the effectiveness of antimalarial drugs. 
The various epidemiological, economical, and other practical aspects regarding the applicability of the test evaluated by us have been shown in Table 5 .

It is concluded that the Flowcytometry based 5 parts differential haematology analyser, is a rapid, high throughput, automated and cost effective device for early detection of malaria. It is capable of detecting specific abnormalities in the blood of patients suffering from malaria in the form of abnormal scattergrams. Detection of malaria by abnormal scattergram in the flowcytometric analyser is not only economical, but can also be easily screened, interpreted as well as documented without any aid of specific technical expertise. Therefore, it is advisable that all laboratory personnel dealing with 5 part differential haematology analysers should be aware of this aspect of abnormal malaria scattergram, so that an early and prompt diagnosis of malaria can be made, even in unsuspected malaria cases. The present study also given advice incorporation of abnormal scattergram events formed in malaria cases as "malaria flags" into modern analysers which will help in early screening and detection of malaria both in endemic and non-endemic regions.

\section{References}

Ben Ezra, J., St. Louis, M., and Riley, R. 2001. Automated malarial detection with the Abbott Cell-Dyn 4000 hematology analyser. Laboratory Hematology. 7: 61-64.

Briggs, C., Da Costa, A., Freeman, L., Aucamp, I., Ngubeni, B., and Machin, S.J.2006.Development of an automated malaria discriminant factor using VCS technology. Am J Clin. Pathol. 126 (5): 691-8.

Campuzano-Zuluaga, G., Hänscheid, T., and Grobusch, M. P. 2010. Automated haematology analysis to diagnose malaria. Malaria Journal, 9, 346.

Enosolease, M.E., and Awodu, O. A. 2003. Seasonal variation of malaria parasitaemia in an Urban Tropical City. Nigeria. J. Clin. Pract. 6(1): 30-33.

Hanscheid, T. 1999. Diagnosis of malaria: a review of alternatives to conventional microscopy. Clin Lab Haematol. 21(4): 235-45.

Hanscheid, T., Melo-Cristino, J., and Pinto, B.G. 2001. Automated detection of malaria pigment in white blood cells for the diagnosis of malaria in Portugal. Am J Trop Med Hyg. 64:290-92.

Huh, H.J., Oh, G.Y., Huh, J.W., and Chae, S.L. 2008. Malaria detection with the Sysmex XE-2100 hematology analyser using pseudoeosinophilia and abnormal WBC scattergram. Ann Hematol. 87 (9):755-9.

Jamaiah, I., Rohela, M., Nissapatorn, V., Khoo, B.L., Khoo, P.S., Radhiyah, M., and Aisyah, A. 2005. Malaria: a 10year (1994-2003) retrospective study at University Malaya Medical Center (UMMC), Kuala Lumpur, Malaysia. Southeast Asian J Trop Med Public Health. 36 Suppl 4:60-3.

Kain, K.C., Harrington, M.A., Tennyson, S., Keystone, J.S.1998. Imported malaria: prospective analysis of problems in diagnosis and management. Clin Infect Dis. 27 (1): 142-9.

Levine, R.A., Wardlaw, S.C., and Patton, C.L. 1989. Detection of haematoparasites using quantitative buffy coat analysis tubes. Parasitol Today. 5 (4): 132-34.

Madhavan, K.T., Jajoo, U.N., and Bhalla, A. 2001. Seasonal variations in incidence of severe and complicated malaria in central India. Indian J Med Sci. 55 (1):43-6.

Moore, C.S., and Cheong, I. 1995. Audit of imported and domestic malaria cases at 
Kuala Lumpur Hospital. Br J ClinPract. 49 (6):304-7

Snounou, G., Viriyakosol, S., Jarra, W., Thaithong, S., and Brown, K.N. 1993. Identification of the 4 human malaria parasite species in field samples by the polymerase-chain reaction and detection of a high prevalence of mixed infections. MolBiochemParasitol. 58 (2): 283-292.

Tangpukdee, N., Duangdee, C., Wilairatana, P., and Krudsood, S. 2009. Malaria Diagnosis: A Brief Review. Korean J Parasitol. 47 (2): 93-102.

Thomson, M.C., Connor, S.J., Phindela, T., and Connor, S.J. 2005. Rainfall and seasurface temperature monitoring for malaria early warning in Botswana. Am J Trop Med Hyg. 73:214-221
Yoo, J.H., Song, J., Lee, K.A., Sun, Y.K., Kim, Y.A., Park, T.S., and Choi, J.R. 2010. Automated detection of malariaassociated pseudoeosinophilia and abnormal WBC scattergram by the Sysmex XE-2100 hematology analyser: a clinical study with 1,801 patients and real-time quantitative PCR analysis in vivax malaria-endemic area. Am J Trop Med Hyg. 82: 412-414.

Zalis, M.G., Ferreira-da-Cruz, M.F., Balthazar-Guedes, H.C., Banic, D.M., Alecrim, W., Souza, J.M., Druilhe, P., and Daniel-Ribeiro, C.T. 1996. Malaria diagnosis: standardization of a polymerase chain reaction for the detection of Plasmodium falciparum parasites in individuals with low-grade parasitemia. Parasitol Res. 82:612-616

\section{How to cite this article:}

Arti Negi, Bijay Ranjan Mirdha, S. Arulselvi, Avinash Singh, Sangram Singh Patel, Anjali Agarwal and Jyotish C. Samantaray. 2018. Evaluation of an Automated, High Throughput Flowcytometry Based Technique for Diagnosis of Malaria. Int.J.Curr.Microbiol.App.Sci. 7(06): 941-951. doi: https://doi.org/10.20546/ijcmas.2018.706.112 